\title{
Gestational Trophoblastic Disease Spectrum: Case Report and Anaesthetic Implications
}

\author{
Vennila Rajagopal ${ }^{1}$
}

\section{Abstract}

Gestational Trophoblastic Disease (GTD) is a spectrum of tumours with abnormal trophoblastic proliferation. The clinical presentation is often with abdominal pain, mild to severe bleeding with or without symptoms due to metastases. Symptoms vary based on the organ of metastasis like breathlessness and cough with chest involvement, lethargy, loss of memory or seizures with brain metastasis. It may range from being pre-malignant conditions like hydatidiform mole and partial hydatidiform mole or be neoplastic invasive mole, choriocarcinoma and rare type epitheloid trophoblastic tumour. There's a huge variation in the incidence of GTD with ethnicity and geography, quoted as high as 1 in 400 pregnancies in Asia as opposed to 1 in 1500-2000 pregnancies in Europe and the United States. Following a molar pregnancy, risk of further GTD increases to $1 \%$ and with more such gestations risk increases by 15-20\%. Undetected hyperthyroidism can comlicate GTD and manifestations of the disease are attributed to excess secretion of human chorionic gonadotropin (HCG) that has thyrotropic activity due to its structural similarity. GTD occurs in 1:40,000 pregnancies and is reported to be more common in Asia. They have the potential to perforate the uterine wall, metastasize and may even lead to death if not treated appropriately. Management of patients with GTD has to be tailored to every patient, following through history taking, examination and investigation. Multisystem involvement with extensive disease can be challenging in the perioperative situation and hence as anaesthetists it becomes important for us to know the pathophysiology, clinical presentations and potential complications. We hereby report two cases in different areas of the disease spectrum and how we managed them.

Keywords: Molar pregnancy; Choriocarcinoma; Tropoblastic disease.

\section{Introduction}

Gestational Trophoblastic Disease (GTD) is a spectrum of tumours with abnormal trophoblastic proliferation. The clinical presentation is often with abdominal pain, mild to severe bleeding with or without symptoms due to metastases. Symptoms vary based on the organ of metastasis like breathlessness and cough with chest involvement, lethargy, loss of memory or seizures with brain metastasis. It may range from being pre-malignant conditions like hydatidiform mole and partial hydatidiform mole or be neoplastic invasive mole, choriocarcinoma and rare type epitheloid trophoblastic tumour $[1,2]$. There's a huge variation in the incidence of GTD with ethnicity and geography, quoted as high as 1 in 400 pregnancies in Asia as opposed to 1 in 1500-2000 pregnancies in Europe and the
United States. Following a molar pregnancy, risk of further GTD increases to $1 \%$ and with more such gestations risk increases by 15 $20 \%[3,4]$. Undetected hyperthyroidism can comlicate GTD and manifestations of the disease are attributed to excess secretion of human chorionic gonadotropin (HCG) that has thyrotropic activity due to its structural similarity $[3,4]$. GTD occurs in 1:40,000 pregnancies and is reported to be more common in Asia. They have the potential to perforate the uterine wall, metastasize and may even lead to death if not treated appropriately [5]. Management of patients with GTD has to be tailored to every patient, following thorough history taking, examination and investigation. Multisystem involvement with extensive disease can be challenging in the perioperative situation and hence as anaesthetists it becomes important for us to know the pathophysiology, clinical presentations and potential complications. We hereby report two cases in different areas of the disease spectrum and how we managed them.

Case Report

\section{Case 1}

A 30 years old lady, weighing $64 \mathrm{~kg}, 165 \mathrm{~cm}$ tall, G3P0A2L0 presented with history of amenorrhoea for three months and intermittent spotting. She had no other significant past medical or surgical history. She was diagnosed to have partial molar pregnancy, with $\beta$ HCG (Human Chorionic Gonadotrophin) levels $>200,000 \mathrm{mIU} / \mathrm{ml}$. She had undergone cervical dilation and endometrial curettage $(D \& C)$ few weeks ago at a different hospital and had continued to have per vaginal (PV) bleeding and hence referred to our centre. Ultrasound scanning at

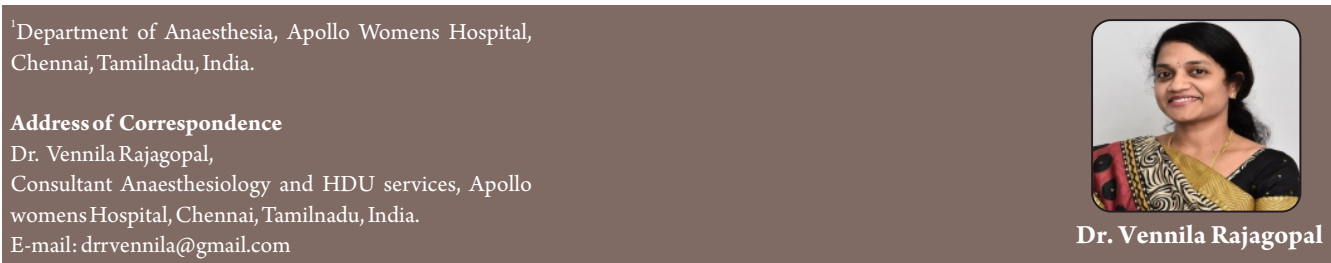

(C) 2020 by Journal of Anaesthesia and Critical Care Case Reports| Available on www.jaccr.com | DOI: 10.13107/jaccr.2020.v06i03.162

This is an Open Access article distributed under the terms of the Creative Commons Attribution Non-Commercial License (http://creativecommons.org/licenses/by-nc/3.0) which permits unrestricted non-commercial use, distribution, and reproduction in any medium, provided the original work is properly cited. 


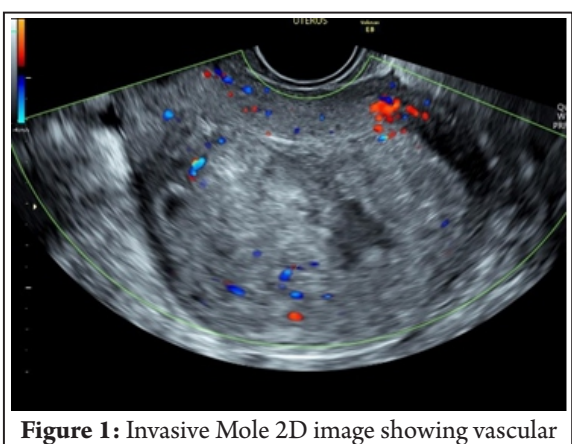

Figure 1: Invasive Mole 2D image showing vascular lesion invading myometrium.

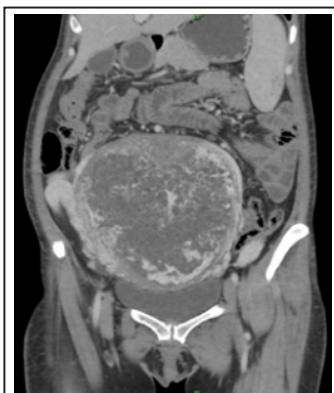

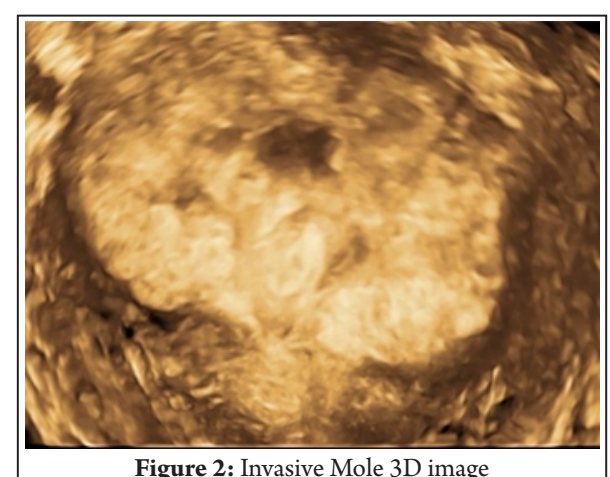

Figure 2: Invasive Mole 3D image

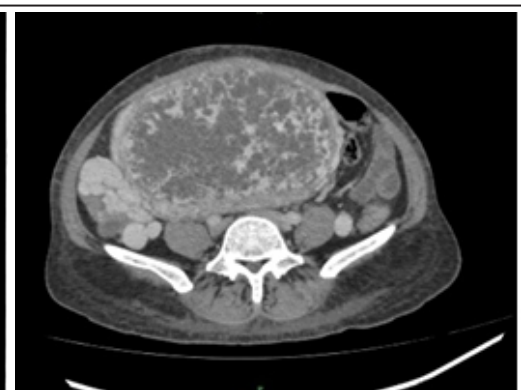

Figure 3,4,5: CT images in different planes our unit confirmed the presence of partial molar pregnancy which was invasive in nature. The Ultrasound images (2D and 3D) are attached in figures 1 and 2 respectively.

On clinical examination, she was hemodynamically stable and respiratory system examination was unremarkable. Renal and liver functions on investigation were unremarkable too. Her complete blood counts revealed haemoglobin of $6.8 \mathrm{gm} / \mathrm{dl}$, other haematological and coagulation parameters being normal. Patient was listed for ultrasound guided evacuation of products of conception under general anaesthesia, with a back-up plan for progression to laparotomy. Informed consent was obtained for the same. Adequate blood products were reserved in anticipation of bleeding complications. Surgical team included an experienced gynaecologist and a vascular surgeon, keeping in mind the potential need for a laparotomy to arrest excessive uterine bleeding from major pelvic feeders. Patient was provided with a detailed explanation of the plan and informed consent was obtained.

Prior to induction of anaesthesia, two wide bore 16-gauge cannulae were secured. Noninvasive monitors as per ASA standards were established. In addition to minimum standard monitoring; temperature, urine output, invasive blood pressure and blood loss were monitored during surgery. Forced air warmer was applied and warm IV fluids were administered to prevent hypothermia. General anaesthesia (GA) was induced using intravenous agents and airway secured with endotracheal tube. Anaesthesia was maintained with inhalational agents and intravenous multimodal analgesia was administered using a combination of opioids and Paracetamol.

Surgeon commenced the procedure under ultrasound guidance. As the products of conception were manipulated, profuse bleeding began, with a rapid blood loss of approximately a litre. Haemodynamic stability was maintained with rapid infusion of one litre warm crystalloid and two units of packed red blood cells to compensate for the losses. She maintained a mean blood pressure $>70 \mathrm{~mm} \mathrm{Hg}$, urine output $>1 \mathrm{ml} / \mathrm{kg} /$ hour and heart rate around 95 beats/min with no new ECG changes on the monitor. The procedure was completed vaginally under ultrasound guidance. Haemostasis was confirmed at the end of the procedure. Oxytocin 10 units in $250 \mathrm{ml} 0.9 \%$ normal saline solution was administered at rate of $100 \mathrm{ml} /$ hour intraoperatively. Patient was successfully extubated at the end of procedure and shifted to the high dependency unit. Her complete blood count and coagulation profile were checked at the end of the procedure revealed haemoglobin of $7 \mathrm{gm} / \mathrm{dl}$ with normal coagulation profile. We did not correct this level of anaemia as she was clinically well and hemodynamically stable. She was shifted back to the ward the following day after ensuring that all vital parameters were within normal range. A week later, on investigation her serum $\beta$ HCG levels had dropped to $3240 \mathrm{mIU} / \mathrm{ml}$ suggesting successful management of the case.

\section{Case 2}

A 32 years old nulliparous woman weighing just $45 \mathrm{~kg}$ presented to gynaecology emergency room with history of twelve weeks gestation and continuous pervaginal bleeding. She had been seen at another hospital three weeks ago and diagnosed to have invasive molar pregnancy with hyperthyroidism. There she was commenced on Methotrexate and antithyroid drug Methimazole 20mg twice daily. Despite the treatment, she continued to progressively deteriorate with pervaginal bleeding.

At presentation to our unit, she was pale, breathless, anxious and looked exhausted. Her vital signs revealed heart rate of 98 beats/min, respiratory rate of 28 breaths/ $\mathrm{min}$ and oxygen saturations of $94-95 \%$ on room air. She could however lie flat. Chest auscultation revealed normal breath sounds. There were no eye signs or tremors suggesting clinical hyperthyroidism. Her blood investigations revealed haemoglobin of 6.0 $\mathrm{g} / \mathrm{dl}$, platelets $100 \times 109 / 1$ and $\mathrm{TSH}<$ $0.07 \mathrm{IU} / \mathrm{ml}$. Free T3 was $>400 \mathrm{ng} / \mathrm{dl}$, free T4 $>6 \mathrm{ng} / \mathrm{dl}$ and $\beta \mathrm{HCG}$ was $>12,00,300$ $\mathrm{mIU} / \mathrm{ml}$. The working diagnosis at this stage was metastatic malignant molar pregnancy complicated by hyperthyroidism. Echocardiography revealed normal left ventricular systolic function with no regional wall motion abnormality and left ventricular ejection fraction was calculated at $60 \%$. Chest $\mathrm{X}$ ray revealed bilateral suspicious metastatic lesions. She was further investigated and her Computed Tomography (CT) scans demonstrated invasion of molar pregnancy into the urinary bladder and pelvic wall bilaterally (Figures 3, 4 and 5). CT scan of the chest confirmed multiple metastatic lesions of the lungs. Endocrinologist were involved to further optimise her thyroid status. They advised to add beta-blocker perioperatively if patient had tachycardia and/or tremors. Although biochemically hyperthyroid, she was clinically asymptomatic with response to methimazole treatment.

In view of the complexity of the disease process involved, a multidisciplinary team was involved to ensure appropriate care plan was formulated and followed. As per guidelines issued by American college of Obstetricians and Gynaecologists (ACOG), 
Royal college of Obstetricians and Gynaecologists ( UK) and FIGO (International Federation of obstetrics and gynaecology) for management of metastatic trophoblastic diseases, it was planned that patient will have a course of multidrug chemotherapy followed by radical hysterectomy $[6,7,8]$. Following the first cycle of multidrug chemotherapy she developed an episode of sepsis that required admission to the high dependency care (HDU).

During her stay in the HDU, she received intravenous broad spectrum antibiotic therapy and close titration of fluid balance. She did not require respiratory or vasopressor support. She responded and recovered in a week's time. We were concerned about further development of neutropenia and coagulopathy, but she did not deteriorate. The raised white cell counts, settled at the end of the week to baseline values. Her renal and coagulation functions remained stable during this episode. Her repeat blood investigations revealed haemoglobin of $6 \mathrm{gm} / \mathrm{dl}$ and with other parameters in normal range. The lesion proved resistant to chemotherapy, hence decision to proceed with radical hysterectomy was taken. The surgery was planned a week after her recovery from the septic episode.

A thorough preoperative anaesthetic assessment was done. She was pale, apyrexial and ambulatory. She was mildly tachypnoeic with respiratory rate of 22 breaths/min and mildly tachycardic with heart rate of 96 beats/min and blood pressure of 90/60 $\mathrm{mmHg}$. She was maintaining oxygen saturations of $95 \%$ on room air. Respiratory system examination revealed normal vesicular breath sounds audible in all zones of the lungs. Difficulty in venous access was anticipated.

Her haemoglobin level was stable at $6 \mathrm{gm} / \mathrm{dl}$ and other parameters like platelet counts, coagulation profile, liver and renal functions continued to be within normal limits. Baseline pulmonary function tests and echocardiography demonstrated normal lung and cardiac function at rest. In summary, we were planning for a radical surgery with potential major haemorrhage in a patient with locally invasive trophoblastic malignant disease, which could be surgically challenging and medically complicated by hyperthyroidism (symptomatically controlled with antithyroid drugs).

Perioperative anaesthetic concerns included:
1. anaemia with ongoing blood loss, 2 . symptomatically corrected hyperthyroidism, 3. post chemotherapy 4. post septic episode and 5. radical operative procedure with potential major blood loss. Anaesthesia plan included awake lumbar epidural for perioperative analgesia followed by induction of general anaesthesia. Standard monitoring would be supported with invasive arterial blood pressure and central venous pressure monitoring. Epidural analgesia was included in the plan, having confirmed the absence of any pre-existing coagulopathy primarily to ensure adequate analgesia, eliminate stress response and sympathomimetic trigger in the patient with hyperthyroidism.

To ensure adequate preoptimization, we administered two units packed red blood cells a day before and reserved adequate number of blood products for intra-operative replacement. Antithyroid drugs were continued. It was imperative to ensure strict asepsis and provide broad spectrum antibiotic cover perioperatively. Patient and her relatives were provided with detailed information on surgery, anaesthesia plan, risk of major blood loss with need for transfusion and postoperative intensive care requirement. Informed high risk consent was obtained.

On the day of the surgery as planned, after gaining large bore venous access on the left hand and establishing standard monitoring, lumbar epidural was placed successfully under strict aseptic precautions. Prior to induction of anaesthesia, intravenous Dexamethasone 8mg, Fentanyl $75 \mathrm{mcg}$ and Metoprolol $2 \mathrm{mg}$ were administered to obtund sympathomimetic response to tracheal intubation. General anaesthesia was induced with Propofol $120 \mathrm{mg}$ and muscle relaxant Atracurium 30 mg was used. Airway was secured with endotracheal intubation. Haemodynamic stability was maintained, with no requirement for further betablockade at that stage. Intravenous preparation of Esmolol and Noradrenaline infusions were attached for use in emergency. Arterial line was secured for continuous blood pressure monitoring and blood gas analysis. Central venous cannulation of the internal jugular vein was done for monitoring and access for administration of inotropes if required. Non pharmacological thromboprophylaxis measures with compression stockings and sequential compression device were applied prior to induction of anaesthesia. Urinary bladder was catheterised to assess urine output. Temperature was monitored and, body warming and fluid warming device were used to prevent hypothermia. We were appropriately prepared to manage 1 . complications related to hyperthyroidism i.e arrhythmias and/or thyroid storm and 2 . complications arising from major haemorrhage.

Midline laparotomy was performed to access the tumour. Intraoperatively, extensive neovascularisation of the tumour led to profuse rapid bleeding. Blood loss was replaced with infusion of warm Ringer's lactate solution and blood products. The patient underwent radical hysterectomy with partial bladder resection and pelvic clearance. During the surgery, she received four units packed red blood cells and four units of fresh frozen plasma. Blood loss was estimated to be around three litres. Along with volume replacement, low dose noradrenaline infusion was started to maintain mean arterial pressure $>60 \mathrm{mmHg}$. Intravenous Tranexamic acid $1 \mathrm{gm}$ was administered to reduce blood loss. At the end of the procedure that lasted for four hours, patient was hemodynamically stable and acid base status was maintained within normal range. Hourly urine output was greater than $0.7 \mathrm{ml} / \mathrm{kg} / \mathrm{hr}$. Her haemoglobin value was $6.5 \mathrm{gm} / \mathrm{dl}$, platelets $110 \times 109 / 1$ and coagulation parameters were within normal range. Serum fibrinogen level was $3.4 \mathrm{~g} / \mathrm{dl}$. Another unit of packed red blood cells was administered to achieve a $\mathrm{Hb}$ of $>7 \mathrm{gm} / \mathrm{dl}$. It would have been ideal use point of care testing of coagulation, but due to lack of access, we did the gold standard laboratory tests. Serum fibrinogen level was acceptable and patient's coagulation tests were within normal limits. We did not transfuse further blood products as per the transfusion recommendations in major obstetric haemorrhage as her Fibrinogen levels were found to be within normal range [9]. We were cautious to prevent fluid overload and transfusion relation lung injury. At the end of the surgery, neuromuscular blockade was reversed and she woke up with good respiratory efforts. Tracheal extubation was performed at the end of the procedure and patient was shifted to the HDU for postoperative care and monitoring. Broad spectrum intravenous antibiotic therapy, a n t ithyroid medications and thromboprophylaxis were continued into the post-operative period. Her recovery following the surgery was uneventful, she did not require any inotropic or respiratory 
support. She was closely monitored for her susceptibility to develop arrythmias, coagulopathy and embolic events. Epidural was removed 48 hours after the surgery, timing the removal 12 hours following the last dose of prophylactic low molecular weight heparin. Two days later, her $\beta$ HCG was checked and demonstrated a downward trend and the TSH levels were also climbing towards normal range.

A week later, her $\beta$ HCG was $8000 \mathrm{mIU} / \mathrm{ml}$ and TSH $0.66 \mathrm{IU} / \mathrm{ml}$. The histopathological report confirmed the diagnosis of an invasive mole, gestational trophoblastic neoplasia with metastasis. She was referred to the oncology team for chemotherapy. One year down the line, she remains asymptomatic.

\section{Discussion}

GTD is a spectrum of premalignant conditions that are characterised by abnormal proliferation of placental trophoblast with secretion of excessive $\beta \mathrm{HCG}$, including hydatidiform molar pregnancy which can be either complete or partial. Molar pregnancies develop as a result of abnormal fertilisation. HCG has alpha and beta subunits. The alpha being identical to TSH (Thyroid stimulating hormone), leads to hypersecretion of thyroid hormones. The serious complications associated with this condition can be due to secondary thyrotoxicosis, high cardiac output failure, arrhythmias, major haemorrhage, coagulopathy, pulmonary artery embolism, disseminated intravascular coagulation, and pulmonary oedema (may also be related to anaemia) $[10,11]$. The malignant forms are collectively called Gestational Trophoblastic Neoplasia (GTN). GTD occurs in 1:40,000 pregnancies and is reported to be more common in Asia. There are huge variations in the incidence of GTD in relation to ethnicity and geography, quoted as high as 1 in 400 pregnancies in Asia as opposed to 1 in 1500-2000 pregnancies in Europe and the United States. Following a molar pregnancy, risk of further GTD increases to $1 \%$ and with more such gestations risk increases by $15-20 \%[12,13]$.

Patients with GTD usually present with vaginal bleeding but they may also present with symptoms of preeclampsia, hyperemesis and hyperthyroidism due to excessive HCG levels in the blood. Very rarely, they present with haemoptysis or seizures due to metastatic disease. Hyperthyroidism is not uncommon in molar pregnancy, but complication with thyroid storm due to lack of control of thyroid status is of rare occurrence, incidence quoted to be around 2$4 \%$ of all pregnancies $[14,15]$. They are often listed for urgent or emergency suction and evacuation of the products of conception.

Recent update on Royal College Obstetricians and Gynaecologists green top guideline on management of GTD was published in September 2020. They have recommended surgical evacuation of gestational trophoblastic disease under ultrasound guidance as the ideal treatment. Anti D prophylaxis and Oxytocin are recommended following complete removal of molar tissue.

In case of metastatic disease, they must be treated with single or multiagent chemotherapy, based on FIGO 2000 scoring system. American college of Obstetricians and Gynaecologists (ACOG), Royal college of Obstetricians and Gynaecologists (UK) and FIGO (International Federation of obstetrics and gynaecology) charted the evidence-based guidelines for diagnosis and management of GTD to prevent morbidity. They provide clear indications for chemotherapy based on the FIGO scoring system. The indications include; 1 . Plateaued or rising $\beta \mathrm{HCG}, 2$. Heavy bleeding wither vaginally or gastro intestinal, 3 . Histological evidence of choriocarcinoma and 4. Evidence of metastasis. Chemo resistant lesions will require surgical treatment. Prognosis for GTN is generally very good if identified and treated on time. Hyperthyroid state may exist for long after removal of molar tissue and follow up monitoring of thyroid hormone levels may be needed. Follow up of the patient with serial $\beta H C G$ levels and that of the histopathological report is important to guide further management for such patients and prevent greater morbidity.

Anaesthesia management must be guided stepwise: systematic preoperative evaluation, preoptimization, planning and conduct of anaesthesia and postoperative care.

Foremost, systematic preoperative evaluation of the patient is essential. Anaesthetic implications in patients with this disease depend on multiple factors. They can be classified as, 1. Lesion related- extent of the lesion, adjacent organ involvement, anticipated blood loss, coagulopathy, thrombotic complications, 2. Thyroid involvement with potential for intraoperative thyroid storm, arrhythmias, high output cardiac failure 3 . Other system involvement, cardiovascular, pulmonary, renal and hepatic 4. Metastatic space occupying lesions (SOL), symptomatic or nor, for example SOL in the brain. According to FIGO scoring system for GTN, risk factors such as age, antecedent pregnancy, pretreatment HCG, tumour size, site and number of metastasis and history of failed chemotherapy helps to decide high risk versus lower risk category. 95\% are low risk cases as defined by FIGO scoring system.

Secondly, preoperative optimisation is of paramount significance. Our patient had responded well to antithyroid drugs and was in a relatively asymptomatic state. She did not require any betablockade in the perioperative period. In terms of other systems involvement, they were maintained near normal without serious sequelae. However, anaemia related myocardial ischemia, cardiac failure, pathology related thromboembolic phenomena, coagulopathy, sepsis, hyperthyroid status with risk of thyroid storm were potential anticipated complications. Presence of coagulopathy would have negated the possibility of regional analgesia, particularly with history of recent sepsis. As early as 1955, first case of molar pregnancy complicated by hyperthyroidism was reported by Tisne et al [16]. Evidence suggests that in $67 \%$ of patients, $\beta$-HCG levels greater than $2,00,000 \mathrm{mIU} / \mathrm{ml}$ have been found to suppress TSH (lower or equal to 0.2 $\mathrm{mIU} / \mathrm{ml}$ ), while levels above 4,00,000 m $\mathrm{IU} / \mathrm{ml}$ can lead to suppression in up to $100 \%$ of cases [17]. The clinical implication is that greater the $\beta$-HCG levels, greater the TSH suppression leading to hyperthyroid events. Patients may not always be clinically symptomatic with raised thyroid hormones. However, they do have the risk of thyrotoxicosis [18]. Symptoms like weight loss, easy fatiguability, increased sweating, palpitations, tremors, enlarged thyroid gland and eye symptoms may be present, suggesting clinically significant hyperthyroidism. Biochemical hyperthyroidism is common in a large majority of patients. Due to short duration of action of increased hormonal levels, overt clinical features of hyperthyroidism may not be present. Undetected hyperthyroidism can lead to severe complications during surgery due to 
thyroid storm and may be catastrophic. Hence it is important to check thyroid hormone levels in patients with GTD to prevent serious consequences [19].

Treatment of hyperthyroid status is an important entity and careful titration of therapy needs to be done. Whilst some patients require single drug treatment with beta blockers, others may require multidrug therapy with antithyroid drugs, steroids and Lugol's iodine. Beta blockers are the first choice when rapid correction is required, like in advanced stages, in preparation for surgery and intraoperatively. Gestational trophoblastic disease complicated by hyperthyroidism treated with methotrexate, antithyroid drugs, steroids and betablockers for three weeks prior to surgery was reported by Khanna et al. There is no clear evidence to suggest a specific time interval between medical management and surgery. It is often decided by the clinical condition of the patient and response to antithyroid drugs and chemotherapy. It is advised to avoid delay in surgery in order to control hyperthyroidism in this group of patients. Increased morbidity and mortality have been reported in delaying surgery $[20,21]$.

Guidelines on management of thyroid storm published by the Japan Thyroid Association and Japanese endocrine society in 2016 recommended a multimodal approach with antithyroid drugs, corticosteroids, beta adrenergic blockers and antipyretic agents [23]. Plasmapheresis has been suggested to remove thyroid hormones in circulation on rare occasions when immediate control had been required. Case report of use of plasmapheresis in management of patient with molar pregnancy and thyroid storm have been published [24].

Following preoperative assessment and optimisation, adequate planning for anaesthesia and surgery must be done. Intraoperative concerns included: 1 . Hyperthyroidism related hypermetabolic state, hypertension, cardiac arrhythmias and/or thyroid storm. 2. Blood loss, fluid balance, blood product transfusion, transfusion related lung injury, fluid overload and hemodynamic instability. Preparedness with appropriately skilled surgical and anaesthetic team, equipment, invasive lines, blood and blood products is required. Choice of anaesthetic technique must be guided by patient's condition, nature and length of surgery and potential complications. Case reports suggest that different types of anaesthetic techniques have been used in this category of patients, including neuraxial anaesthesia, general anaesthesia inhalational based and total intravenous anaesthesia to control sympathetic hyperactivity. Ertuk et al published case report of the procedure done with intravenous remifentanil and propofol with esmolol boluses in a patient with hydatidiform mole and hyperthyroidism. Cekic et al suggested regional anaesthesia for suction and evacuation treatment of molar pregnancy with thyrotoxicosis because of tocolytic effect of halogenated agents [25]. The choice we make must be an informed one with a backup for full general anaesthesia and resuscitation facilities being available. The ultimate goals of anaesthetic management are to prevent thyroid storm and excessive sympathetic activity. A large percentage of cases of GTD are low risk and hence we may be able to conduct anaesthesia safely without any adverse events. In case of GTN, preparation is important and caution must be exercised.

Management of major haemorrhage includes preparedness with good intravenous access, blood products, inotropes/vasopressors and good surgical team to arrest the bleeding. We need to maintain haemodynamic stability and ensure end organ perfusion with timely resuscitation until surgical haemostasis is achieved. Application of principles of management of major obstetric haemorrhage allows for efficient management.

As per the guidelines suggested, 1. Maintain homeostasis to ensure adequate cellular respiration, acid base balance, normotension or permissive hypotension $(20 \mathrm{mmHg}$ less than normal) until surgical haemostasis is achieved, normoxia, normocarbia, normothermia, normal electrolyte levels particularly calcium 2. Administer tranexamic acid $1 \mathrm{gm}$ bolus if blood loss $>$ one litre, 3. Transfuse packed red blood cells (PRBC) to replace the loss, when blood loss is significant or when haemoglobin less than $7 \mathrm{gm} / \mathrm{dl}, 3$. Once massive loss is recognised, blood products Fresh frozen plasma and cryoprecipitate may be required, 4 . Transfuse platelets when platelet count $<70 \times 109 / \mathrm{L}, 5$. Cryoprecipitate or fibrinogen concentrates when fibrinogen less than $2 \mathrm{~g} / \mathrm{l}$.

Over the years evidence has accumulated that shows, transfusion of FFP and Cryoprecipitate do not raise fibrinogen levels to the degree required, as their concentrations are $1-3 \mathrm{~g} / 1$ and $1 \mathrm{~g} / 1$ respectively. To replace fibrinogen, volumes required has the potential to cause harm due to fluid overload. Hence point of care testing (POCT) guided transfusion in ideal. In the absence of POCT, lab tests and clinical acumen have to be applied and transfusion protocol needs to be followed. Presence of institutional guidelines for such emergencies will help prevent delays and catastrophes.

Oxytocin infusion and anti-Rhesus D prophylaxis are recommended after complete removal of molar tissue. Postoperatively, close monitoring in critical care unit for acute respiratory distress, cardiac and hemodynamic problems with or without coagulation impairment is required. Multiple case reports have been published with patients developing pulmonary oedema immediately postoperatively or on table with increased surge of catecholamines. When there is an anticipated risk of multiorgan dysfunction, planning becomes essential.

Our second patient, who had GTN with metastasis and hyperthryoidism, which was resistant to chemotherapy belonged to the high-risk category. Clinical management was planned with the multidisciplinary team to enhance successful care. With the patient under combined general and regional anaesthesia, and availability of blood products we were well prepared and able to manage the case safely and efficiently. Postoperatively, she had a smooth course with no hormonal or sympathetic hyperactivity. Two days later, hormone levels began to taper down. Case reports of subsequent increase in hormones and persistent sympathomimetic activity due to remnant tissue or recurrence have been published. Hence follow up for up to one-year period is suggested.

\section{Conclusion}

In conclusion, although rare, molar pregnancies with neoplastic changes and/or metastasis are encountered. This case report is to highlight the importance of a systematic history taking, examination and investigation in these group of patients to assess their risk status and have a perioperative management plan. Appropriate preoperative optimization with antithyroid drugs, vigilant intraoperative anaesthesia care and close monitoring in the postoperative period help to reduce morbidity and mortality in patients with GTD. Ideally, such patients must be managed in a tertiary center with intensive care back up and blood bank facility. 


\section{References}

1. Dave N, Fernandes $S, A m b i$ U, Ayer H. Hydatidiform mole with hyperthyroidism - perioperative challenges. JObstet Gynaecol India 2009;59:356-7

2. Dutta DC. Haemorrage in early pregnancy. In Hiralal Konan, editor. Textbook of Obstetrics. 6 th edition. Kolkatta:New Central BookAgency;2004.p.159-202

3. Seckl MJ, Sebire NJ, Berkowitz RS. Gestational trophoblastic disease. Lancet 2010;376:717729.

4. MJ Seckl, NJ Sebure, RA Fisher, F Golfeir, L Massuger, C Sessa Gestational trophoblastic disease. ESMO clinical practice guidelines. Ann Oncol 2013;0240(Suppl 6: vi39-vi50

5. Lurain, JR. Gestational trophoblastic disease I: epidemiology, pathology, clinical presentation and diagnosis of gestational trophoblastic disease, and management of hydatidiform mole. American Journal of Obstetrics and Gynecology 2010; 203(6):531-539.

6. Khanna P, Kumar A, Dehran M, Gestational trophoblastic disease with hyperthyroidism: Anesthetic management. Journal of Obstetric Anesthesia and critical care 2012;2:31-3

7. Tetsurou Satoh, Osamu Isozaki, Atsushi Suzuki, Shu Wakino, Tadao Iburi, Kumiko Tsuboi, Naotetsu Kanamoto, Hajime Otani, Yasushi Furukawa, Satoshi Teramukai and Takashi Akamizu. 2016 Guidelines for the management of thyroid storm from The Japan Thyroid Association and Japan Endocrine Society (First edition) Endocrine Journal 2016, 63 (12), 1025-1064
8. Azezli A, Bayraktaroglu T, Topuz S, Kalayogly-Besisk S. Hyperthyroidism in molar pregnancy: Rapid preoperative preparation by plasmapheresis and complete improvement after evacuation. Transfus Apher Sci 2007,36:87-9

9. Wonjung Hwang, Daehwan Im, Eunsung Kim, Persistent perioperative tachycardia and hypertension diagnosed as thyroid storm induced by a hydatidiform mole. KlreN Johrnal of anaesthesiology 67(3):205-8

10. Erturk E, Bistan H, Geze S, Saracoglu S, Erciyes N, Eroglu A. Total Intravenous Anaesthesia for evacuation of a hydatidiform mole and termination of pregnancy in a patient with thyrotoxicosis. Int J Obster Anaesth 2007;16:363-6

11.John R Lurain Gestational trophoblastic disease II: classification and management of gestational trophoblastic neoplasia JANUARY 2011 American Journal of Obstetrics \& Gynecology. Oncology Reviews. P 12-18.

12. Celeskj D, Micho J, Walers L Anaesthetic implications of a partial molar pregnancy. and associated complications. AANAJ2001;69:49-53

13. S Swaminathan, Roshin Ann James, Rashmi Chandran, Reesha Joshi. Anaesthetic implications of severe hyperthyroidism secondary to molar pregnancy: A case report and review of literature. Anaesth Essays Res 2017Oct-Dec 2017; 11(4):1115-1117

Acknowledgment: I would like to thank Dr. Kumar G, Consultant Gynae Oncologist, Dr. Shreepriya Sundaram Obstetrician and Sonologist, Dr. Shiv Kumar Singh for their help with this article.

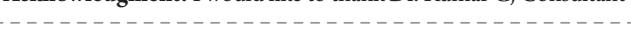

Conflict of Interest: Nil Source of Support: None

\section{How to Cite this Article}

Rajagopal V | Gestational Trophoblastic Disease Spectrum: Case Report and Anaesthetic Implications | Journal of Anaesthesia and Critical Care Case Reports | September-December $2020 ; 6(3): 27-32$. 